\title{
ADHERENCIA A LA DIETA MEDITERRÁNEA EN NIÑOS Y ADOLESCENTES DEPORTISTAS: REVISIÓN SISTEMÁTICA
}

\section{MEDITERRANEAN DIET ADHERENCE IN CHILDREN AND ADOLESCENTS ATHLETES: SYSTEMATIC REVIEW}

\author{
Eva María Peláez Barrios ${ }^{1}$ y Mercedes Vernetta Santana ${ }^{1}$ \\ evapelaezbarrios@gmail.com; vernetta@ugr.es \\ ${ }^{1}$ Departamento de Educación Física y Deportiva, Universidad de Granada, España
}

Envío original: 2020-07-10 Reenviado: 2021-01-15 Aceptado: 2021-01-27

Publicado: 2021-02-04

\section{Doi: https://doi.org/10.15517/pensarmov.v19i1.42850}

\begin{abstract}
RESUMEN
El objetivo de esta revisión fue analizar los estudios realizados en niños y adolescentes deportistas que utilizaban el índice KIDMED para conocer la adherencia a la dieta mediterránea (ADM). Se realizó una búsqueda en diferentes bases de datos WOS, Scopus, Google Scholar, Pubmed y Sport Discuss, entre el 1 de Enero de 2010 y el 1 de Junio de 2020, siguiendo la guía PRISMA. De los 82 estudios identificados, 10 analizaron la ADM y cumplieron con todos los criterios de inclusión. Los resultados obtenidos indicaron que los mayores porcentajes de óptima ADM se evidencian en piragüistas masculinos, seguidos de futbolistas masculino, gimnastas de rítmica y kayakistas femeninas $(66 \%, 54,8 \%, 52,2 \%$ y $51 \%$ respectivamente). En todos los deportistas analizados, los resultados son mejorables, siendo más pronunciada en las jugadoras de futbol sala. En la mayoría de los estudios no existe una relación entre ADM y las medidas antropométricas. Como conclusión se destaca que la mayoría de los deportistas se encuentran entre una mejorable y óptima adherencia a la dieta mediterránea, siendo pocos los que obtienen porcentajes bajos de adherencia a la dieta mediterránea, destacando un patrón de adherencia a la dieta mediterránea aceptable casi en su totalidad pero no óptimo. Además, la mayoría de los estudios incluidos en la revisión sistemática obtienen una alta calidad según las directrices STROBE.
\end{abstract}

Palabras clave: dieta mediterránea, KIDMED, niños, adolescentes, deportistas 


\begin{abstract}
The objective of this review was to analyzed the studies carried out in children and adolescent athletes who used the KIDMED index to know adherence to the Mediterranean diet (ADM). A search was made in different databases WOS, Scopus, Google Scholar, PubMed and Sport Discuss between January $1^{\text {st }}, 2010$ and June $1^{\text {st }}$, 2020, following the PRISMA guide. 82 studies were identified, 10 articles analyzed ADM and complied the inclusion criteria. The results indicated that the highest percentages of optimal ADM are evident in male canoeists, followed by male footballers, rhythmic gymnasts, and female kayakers ( $66 \%, 54,8 \%, 52,2 \%$ and $51 \%$ respectively). In all the athletes analyzed, the results are improbable, being more pronounced in the futsal players. In most studies there is no relationship between ADM and anthropometric measurements. In conclusion, it should be noted that the majority of athletes are between improbable and optimal adherence to the Mediterranean diet, highlighting a pattern of adherence to the Mediterranean diet that is almost acceptable in their whole but not optimal. Furthermore, most of the studies included in the systematic review obtain high quality according to the STROBE guidelines.
\end{abstract}

Keywords: Mediterranean diet, KIDMED, children, adolescents, athletes.

\title{
INTRODUCCIÓN
}

Una dieta óptima con ingestas energéticas adecuadas es uno de los múltiples factores que inciden en el rendimiento deportivo en cualquier tipo de deporte. Además, tener buenos hábitos alimentarios no sólo es importante para conseguir el máximo rendimiento y reducir posibles riesgos de lesiones en los deportistas, sino también, para garantizar la consecución de un estado nutricional óptimo y prevenir problemas de salud en el momento actual y en el futuro (López-Sobaler, Aparicio, y Ortega, 2017).

No obstante, uno de los grandes problemas de este siglo es el cambio de patrones de alimentación desde edades tempranas, agudizándose en la adolescencia por ser una etapa de innumerables cambios tanto físicos como psicológicos incrementándose el consumo de dietas no saludables o las conocidas fast-food (Allen y Waterman, 2019; Lynch et al., 2014).

Se trata de un período que abarca desde los 12 hasta los 21 años, aunque algunos estudios indican un posible aumento en este rango de edad desde los 10 años (preadolescencia) hasta los 24 años (adolescencia tardía), debido a los cambios del estilo de vida que experimentan los jóvenes en la actualidad (Sawyer, Azzopardi, Wickremarathne, y Patton, 2018; Sawyer et al., 2012). 
Varios estudios evidencian que una ingesta insuficiente de energía se asocia con trastornos de alimentación, siendo más frecuente entre las mujeres atletas que en grupos de población normal (Márquez, 2008; Sundgot-Borgen y Torstveit, 2004); dentro de las atletas, son más prevalentes entre las que compiten en deportes dependientes del peso y estéticos como la gimnasia.

La dieta mediterránea es considerada uno de los modelos dietéticos más saludables, con beneficios frente a diferentes enfermedades cardiovasculares 0 incidencia en la mortalidad por cáncer y, por tanto da lugar a una mayor expectativa de vida (Dussaillant, Echevarría, Urquiaga, Velasco, y Rigotti, 2016; Márquez-Sandoval, Bulló, Vizmanos, Cass-Agustench, y Salas-Salvado, 2008; Martínez-González et al., 2008; Rubio-Arias et al., 2015). Tiene la característica de ser una dieta rica en antioxidantes naturales y baja en grasas saturadas, debido al consumo de gran cantidad de legumbres, verduras, frutas, frutos secos, hortalizas entre otros (Martín et al., 2004; Sánchez-Cruz, Jiménez-Moleón, Fernández-Quesada, y Sánchez, 2013; Vernetta, Montosa, Ariza, y López Bedoya, 2019). También incluye aceite de oliva virgen como principal fuente de grasa, cantidades moderadas de pescado y lácteos (básicamente queso y yogurt), con cantidades bajas de carnes rojas. La importancia de este patrón dietético está relacionada con ser una dieta equilibrada y variada que proporciona la mayoría de los macronutrientes recomendados en la proporción adecuada. En definitiva, su característica principal es un bajo contenido en ácidos grasos saturados, así como altas cantidades de fibra y carbohidratos complejos e importantes cantidades de antioxidantes (Donini, Serra-Majem, Bulló, Gil, y Salas-Salvadó, 2015). Por ello, es una dieta muy recomendable para toda la población en general y en particular para los deportistas, ya que gracias a ella el rendimiento de los mismos se puede ver favorecido en un espacio corto de tiempo (Rubio-Arias et al., 2015).

Son varios los estudios que han realizado un análisis de la adherencia a la dieta mediterránea (ADM) en niños y adolescentes (Akar y Sanlier, 2011; Ayechu y Dura, 2010; 2009; Cabrero et al., 2012; Córdoba-Caro, Luego, y García, 2012; Durá-Travé, Gallinas-Victoriano, Mosquera-Gorostidi, Justo-Ranera, y Martín-García, 2014; Santomauro et al., 2014; Zapico, Blández, y Fernández, 2010), pero son pocos los realizados en población deportista (Alacid, Vaquero-Cristóbal, Sánchez-Pato, Muyor, y López-Miñarro, 2014; González-Neira, Mauro-Martín, García-Aguado, Fajardo, y Garicano-Vilar, 2015; Hernández-Camacho, Fuentes-Lorca, y Moya-Amaya, 2017; López Secanell y Rico Mateu, 2019; Manzano-Carrasco et al., 2020; Philippou, Middleton, Pistos, Andreou, y Petrou, 2017; Rubio-Arias et al., 2015; Vernetta, Montosa, y López-Bedoya, 2018; Vernetta et al., 2019). 
En el estudio de Idelson, Scalfi y Valerio (2017) se ha podido observar que hay una gran variedad de trabajos que analizan la adherencia de la dieta mediterránea en niños y adolescentes, pero no se encuentra ninguna evidencia de que exista una recopilación de estudios que se centre en esta población de alguna modalidad deportiva, de ahí que el objetivo del presente estudio fue realizar una revisión sistemática para conocer la ADM en niños y adolescentes que practica alguna modalidad deportiva.

\section{METODOLOGÍA}

Se realizó una búsqueda de artículos publicados en idioma inglés y español en las bases de datos Web of Science, Scopus, Google Scholar, Pubmed y Sport Discuss utilizando los términos MeSH (Medical Subject Headings): "mediterranean diet" AND "kidmed test" AND "adolescents" AND "children" AND "atheletes".

Los criterios de inclusión fueron artículos originales que utilizaran el Cuestionario de Calidad de Vida Mediterránea o índice KIDMED (Serra-Majem, García-Closas, Ribas, Pérez-Rodrigo, y Aranceta, 2001) para medir la ADM, realizados en niños y adolescentes (mujeres y varones) con un rango de edad de 6 a 24 años, practicantes de cualquier disciplina deportiva; publicados en los diez últimos años (desde el 1 enero del 2010 al 1 de Junio de 2020). Se excluyeron trabajos anteriores al año 2010, estudios que fueran revisiones o meta-análisis, artículos con sólo resúmenes publicados, comunicaciones o ponencias presentadas a congresos, tesis doctorales, estudios que incluyera algún tipo de dieta para bajar de peso y todos los trabajos que estuviesen redactados en un idioma diferente al inglés o español.

Se realizaron cinco diferentes búsquedas electrónicas, correspondientes a cada una de las bases indicadas en las cuales se encontraron 82 artículos de los cuales fueron descartados 30 por ser duplicados, quedaron 52 y se realizaron igualmente, búsquedas adicionales utilizando el apellido del primer autor y el término de búsqueda "dieta mediterránea" AND "athletes" en el título, con la detección de referencias recursivas de artículos proyectados (elegidos) para identificar otros artículos posiblemente relevantes. Posteriormente se eliminaron 25 por no encontrarse en el periodo indicado y se revisaron 27 . Tras leer el abstract se eliminaron 10, quedando 17 , de los cuales fueron descartados 7 más por no cumplir los criterios de inclusión. Se analizaron 10 artículos siguiendo las pautas PRISMA para la realización de una revisión sistemática. La figura 1 expone a través de un diagrama de flujo los cuatros pasos siguientes: identificación, detección o artículos proyectados, selección e inclusión (Moher, Liberati, Tetzlaff, y Altman, 2009).

Todo el proceso de selección y evaluación de los artículos se hizo por dos revisoras independientes (EPB; MVS), según las directrices de la Strengthening the 
Reporting of Observational Studies in Epidemiologv (STROBE), para verificar la rigurosidad y calidad técnica de las investigaciones. La lista contiene 22 ítems sobre lo que debe de incluirse en una descripción precisa de los estudios observacionales. Los estudios tienen una puntuación entre cero y 22 puntos. En caso de discrepancias entre las 2 revisoras en la evaluación de algún artículo, se volvía a evaluar hasta llegar al consenso. La puntuación metodológica se comparó con la puntuación máxima de STROBE clasificándose los artículos en tres grupos según su calidad metodológica: calidad alta ( $\geq 70 \%$ de la puntuación total), calidad moderada, (50-69\% puntuación total) y baja calidad ( $<50 \%$ de la puntuación total) (Strong et al., 2005).
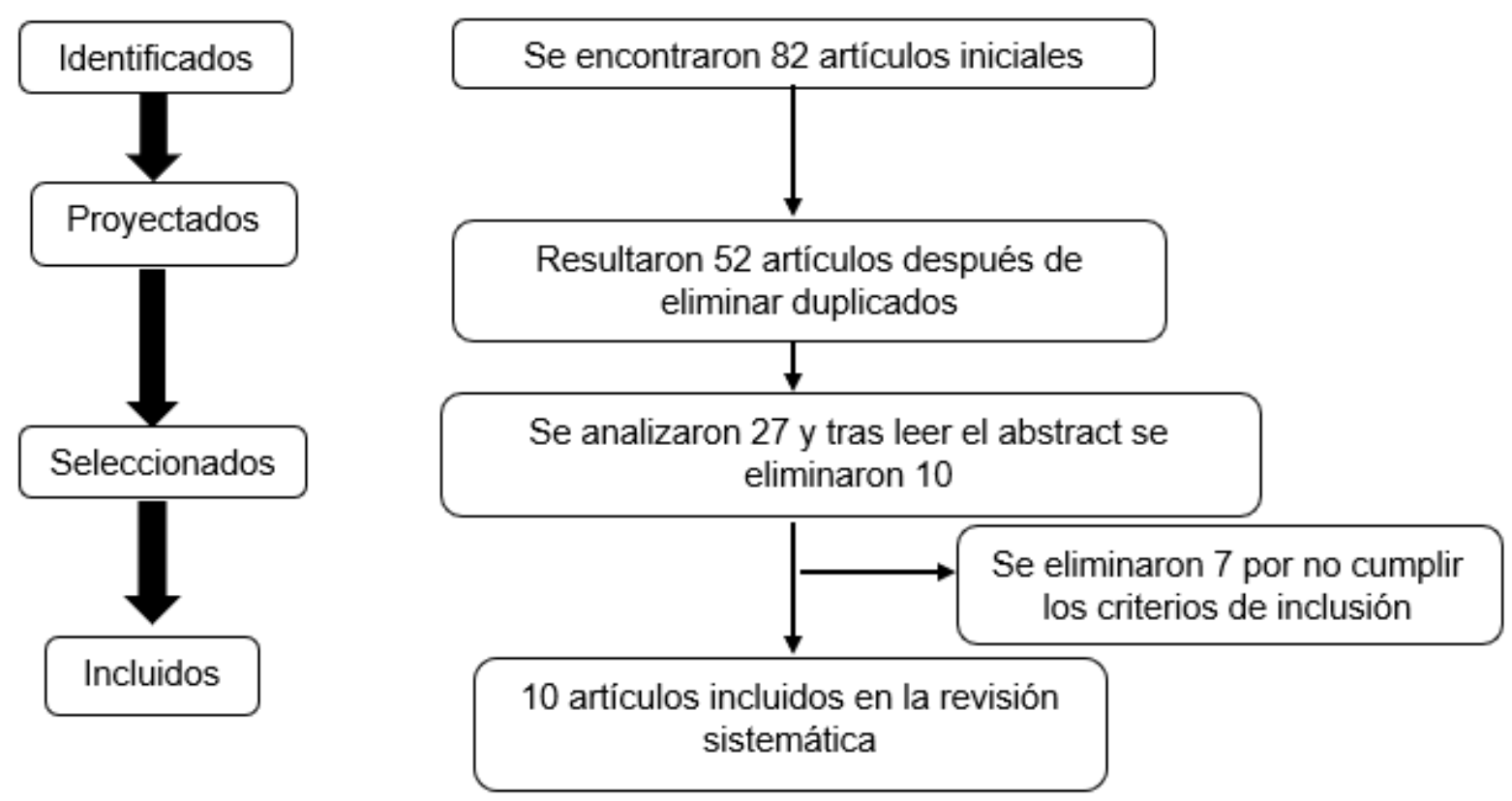

Figura 1. Búsqueda de acuerdo con la metodología PRISMA. Fuente: elaboración propia.

Los estudios fueron codificados en función de una variable contextual teniendo en cuenta dos quinquenios o lustros de los años de publicación de los artículos 2010-2014; 2015-2020.

Para el análisis de datos se registraron los siguientes datos de cada estudio: autores y año, población de estudio (número de participantes, edad, sexo y deporte practicado), calidad metodológica y variables estudiadas, así como resultados obtenidos en cada uno de los estudios. 


\section{RESULTADOS}

En primer lugar, como se observa en la figura 2, de acuerdo con el periodo evaluado (2010 a 2020) las investigaciones de ADM en deportistas son muy escasas en los dos quinquenios. Los años donde existen mayor número de publicaciones son entre el 2015-2020 (9 artículos), experimentando un repunte importante, quizás al incremento y expansión de la preocupación por tener buenos hábitos nutricionales y estilo de vida saludable en los niños y adolescentes deportistas.

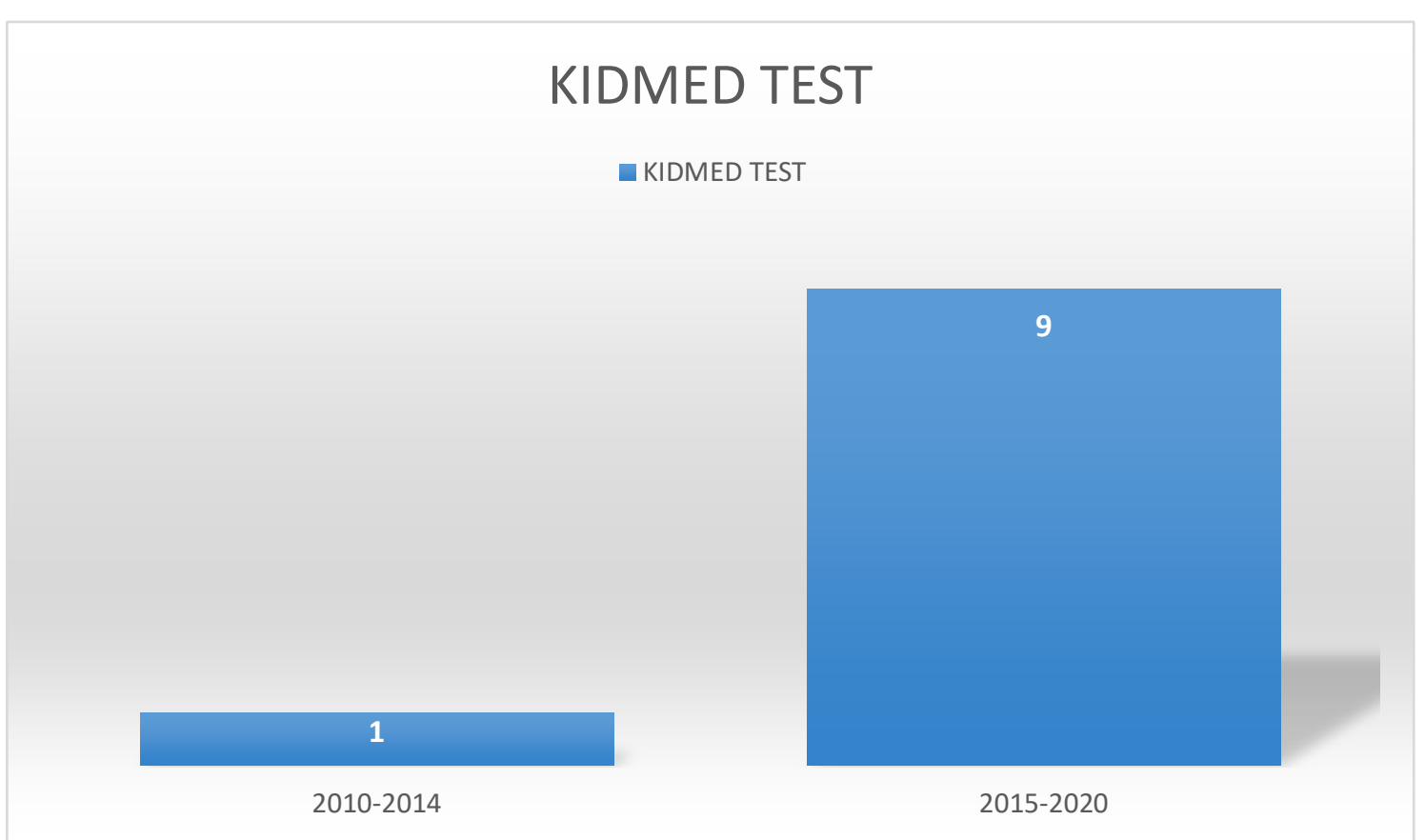

Figura 2. Reparto de publicaciones en los diferentes años analizados. Fuente: elaboración propia.

En general, se evidencia una gran dispersión de publicaciones en diferentes revistas: 2 artículos en la revista Nutrición Hospitalaria y 1 artículo en el resto de revistas: Spanish Journal of Human Nutrition and Dietetic, Revista Andaluza de Medicina Del Deporte, Revista de Transmisión Del Conocimiento Educativo y de La Salud, International Journal of Enviromental Research and Public Health, Journal of Science and Medicine in Sport, MHSalud, Revista Chilena de Nutrición, Revista Brasileira de Medicina Do Esporte.

Los resultados obtenidos de los 10 artículos seleccionados quedan englobados en la Tabla 1. 


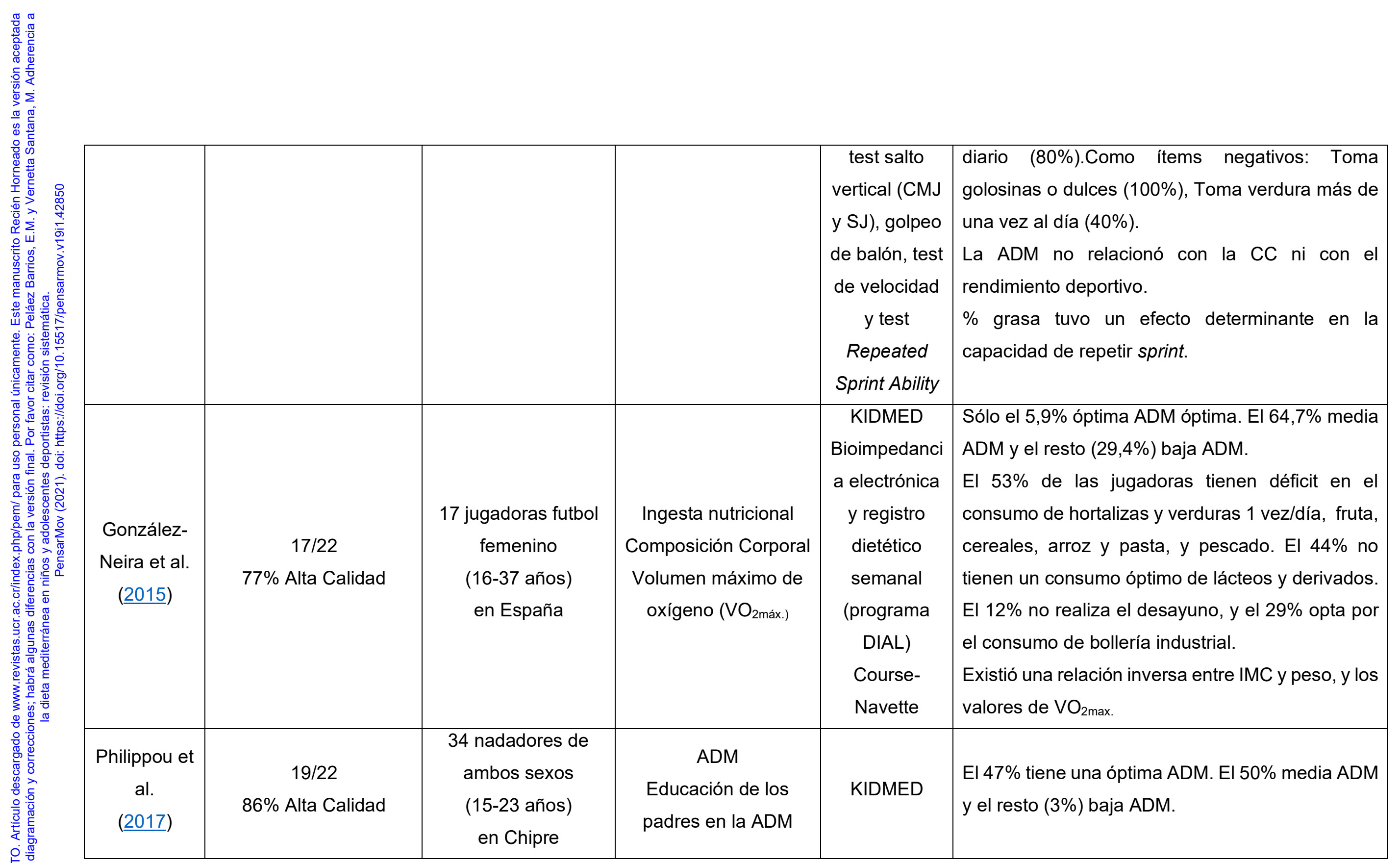




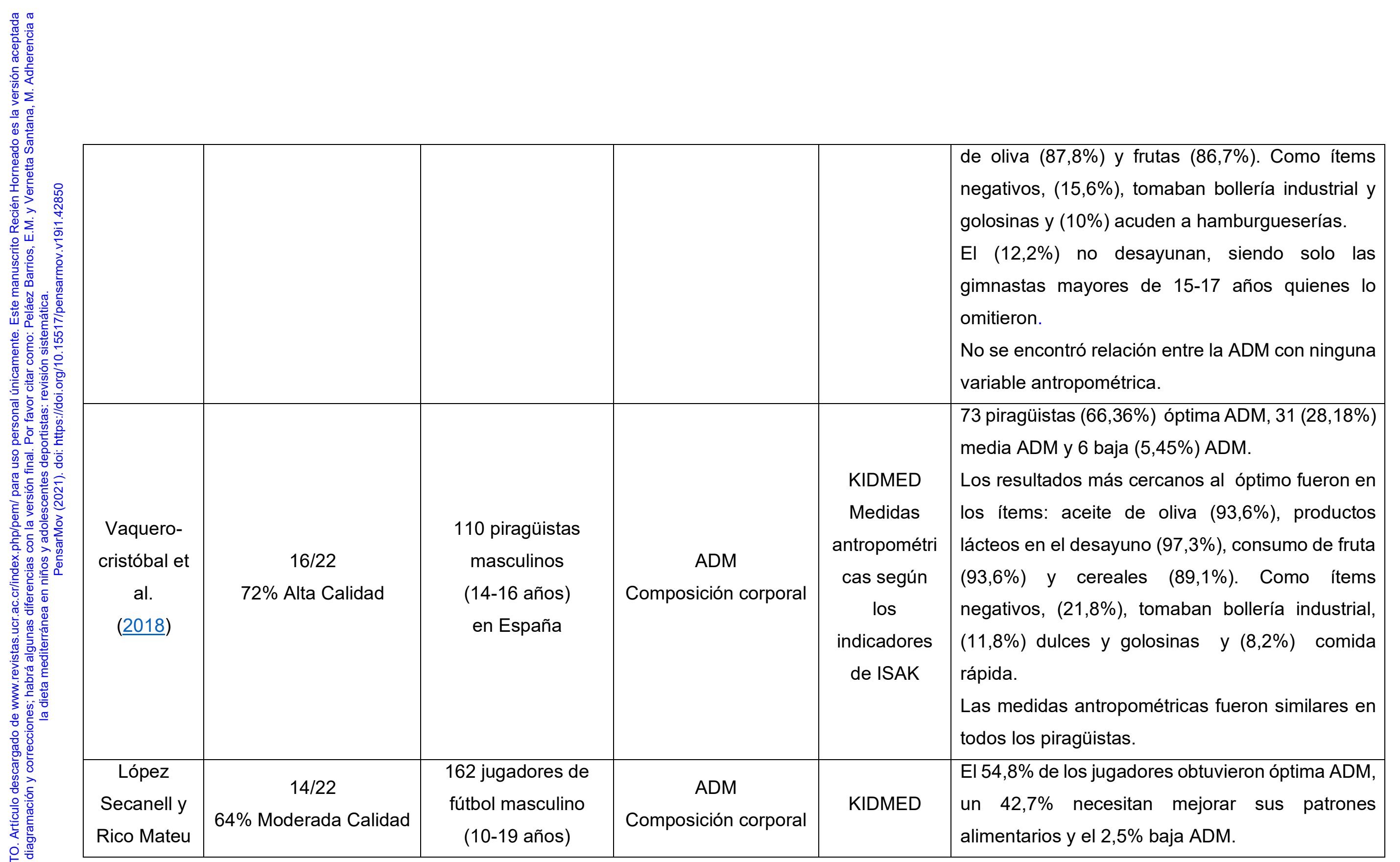




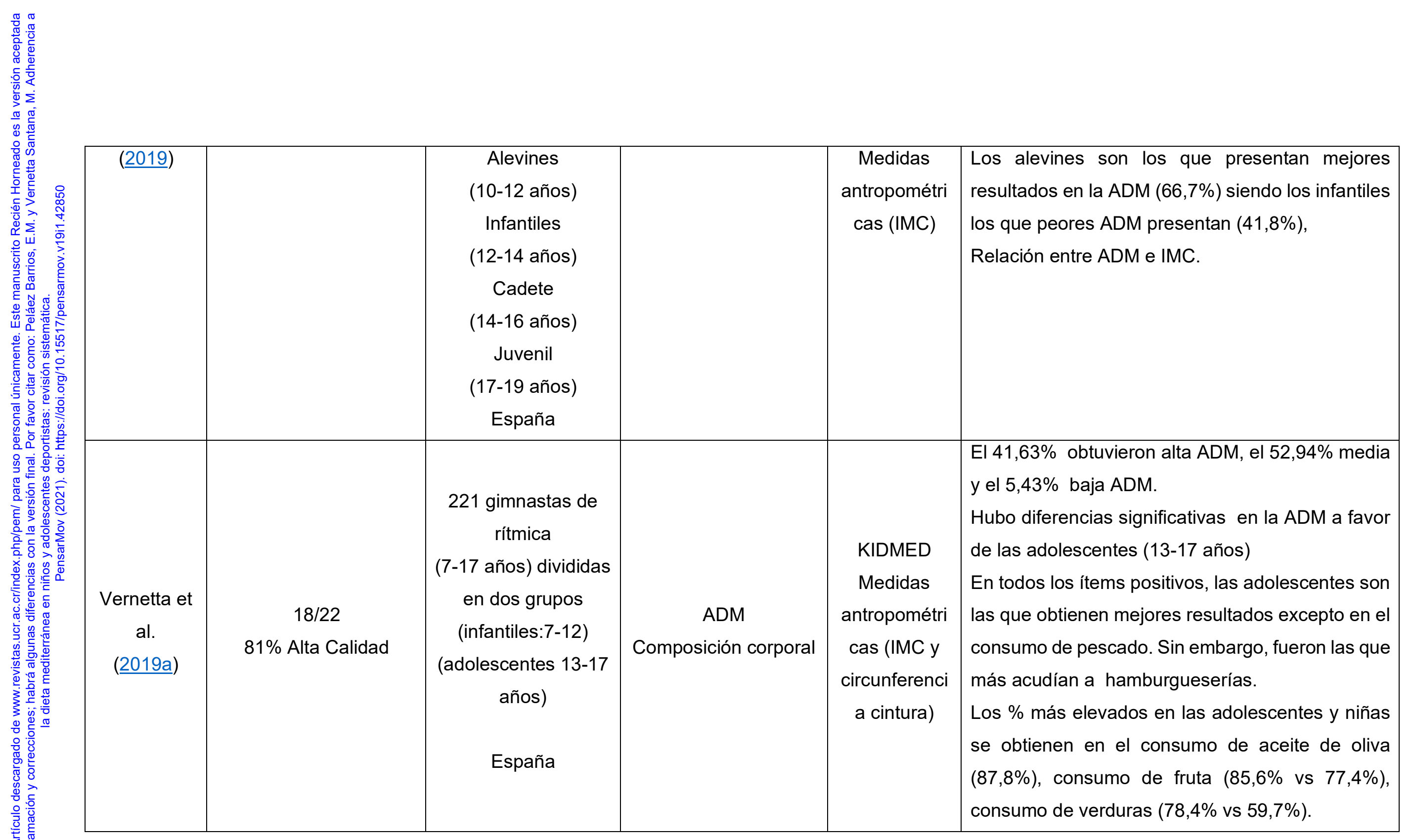




\section{DISCUSIÓN}

Los resultados más destacados en los artículos incluidos en la revisión, indican que la adherencia a la DM en los adolescentes practicantes de alguna modalidad deportiva son mejorables, siendo este aspecto más destacado en jugadoras de fútbol femenino (González-Neira et al., 2015; Rubio-Arias, et al., 2015). En general los deportistas de mayor nivel presentan mejor ADM, predominando el consumo de aceite de oliva, cereales, vegetales y frutas (Vaquero-Cristóbal, Alacid, Muyor, y LópezMiñarro, 2018; Vernetta et al., 2019).

A continuación, la discusión de los datos encontrados en esta revisión se ha organizado en función de los aspectos analizados en los resultados.

\section{Número de autores}

El rango de autores por publicación es de dos a siete, la mayoría de las publicaciones se realizan con la colaboración de cinco autores representando un $30 \%$ (3 artículos), seguido de la colaboración de tres, cuatro y siete autores, representando un $60 \%$ del total (6 artículos), y un único artículo realizado por López Secanell y Rico Mateu $(\underline{2019})$ con dos autores $(10 \%)$.

\section{Tamaño de las muestras, edad y país}

El tamaño de la muestra del total de las investigaciones es variada, oscilando entre 12 deportistas de élite (Rubio-Arias et al., 2015) y 1198 en el estudio de (ManzanoCarrasco et al., 2020), siendo estos deportistas de escuelas deportivas de diferentes deportes y no de élite, de ahí esa gran diferencia.

En relación a la edad, en los estudios analizados se trabajó tanto con niños (6-10 años), preadolescentes (10 a 13 años) como adolescentes (14 a 21 años) incluyendo un solo artículo con adolescentes- adultos (16-37 años), se determinó incluir este artículo, pues solo una de las jugadoras según los autores, tenía 37 años y el resto adolescentes hasta 24 años (González-Neira et al., 2015). Tras analizar los estudios incluidos en la revisión sistemática, 6 artículos $(60 \%)$ han realizado el estudio en población adolescente, 3 estudios (30\%) establecieron el rango de edad con niños y adolescentes y 1 artículo (10\%) ampliaron el rango de edad con adolescentes y adultos (González-Neira et al., 2015), donde el 5,9\% de los encuestados obtuvieron una óptima ADM.

Referente al país de procedencia de la muestra, cabe indicar que el $90 \%$ de los estudios incluidos en la revisión han sido realizados en España, y solo 1 estudio (10\%) fue realizado en Chipre (Philippou et al.,2017). 


\section{Sexo y Tipos de deportes}

El $40 \%$ de los estudios realizados en deportistas han sido en jugadores de futbol tanto de sexo masculino (Hernández-Camacho et al., 2017; López Secanell y Rico Mateu, 2019) como femenino (González-Neira et al., 2015; Rubio-Arias et al., 2015), seguido de los estudios realizados en gimnastas de rítmica (20\%) (Vernetta et al., 2018; Vernetta et al., 2019). Un $30 \%$ se reparte en un estudio con nadadores, uno con practicantes de kayak y otro con piragüistas (Alacid et al.,2014; Philippou et al., 2017; Vaquero-cristóbal et al., 2018). Por último, el 10\% restante, es de un estudio, realizado con deportistas españoles de diferentes escuelas deportivas municipales de Castilla la Mancha (Manzano-Carrasco et al., 2020).

La mayoría de los estudios se han desarrollado en mujeres (50\%), 3 estudios son desarrollados en varones (30\%) y 2 estudios fueron realizados en ambos sexos (20\%). El hecho de haber más estudios con muestras femeninas que con muestras solo masculinas, podría ser debido a que suelen ser las chicas las que más interés y preocupación tienen por tener una buena alimentación y estilo de vida saludable (Moreno y Ortiz, 2009).

\section{Metodología (variables)}

La mayoría de los estudios incluidos en esta revisión (70\%) han obtenido una alta calidad tras haberse analizado con las directrices STROBE, es decir la puntuación obtenida ha sido mayor o igual al 70\%, 3 estudios (30\%) han obtenido una moderada calidad, no encontrándose ningún estudio con baja calidad según estas directrices.

Los resultados de esta revisión indican que ninguno de los estudios analizados (100\%), estudian como única variable la adherencia a la DM, siendo el $40 \%$ de los artículos los que analizan esta variable con la composición corporal (4 artículos), 2 artículos estudian la relación de la adherencia a la DM junto con la actividad física y el rendimiento deportivo, y otros artículos estudian el somatotipo junto con la adherencia a la DM. Igualmente de los 10 estudios que se incluyeron para esta revisión, 1 artículo es longitudinal (10\%) (Philippou et al., 2017) y el resto (90\%) son transversales. Los transversales son de diferente estilo, ya que algunos son descriptivos, cuasiexperimentales, analítico $\mathrm{u}$ observacional.

Todos los estudios analizados (100\%), utilizan el KIDMED para conocer el grado de adherencia a la DM que presentan los deportistas. Para el resto de las variables que se analizan en estos documentos se utilizan otros instrumentos como el PDM, test isocinético, test salto vertical (CMJ y SJ), golpeo de balón, test de velocidad y test Repeated Sprint Ability (RSA) (1 artículo), Bioimpedancia electrónica (BIA), registro 
dietético semanal (programa DIAL) y Course-Navette (1 artículo), y la batería ALPHA (1 artículo).

\section{Resultados encontrados}

En relación al tipo de deporte practicado, los estudios muestran resultados diferentes. Los mayores porcentajes de óptima ADM se evidencia en los piragüistas con un 66\% de adherencia (Vaquero-cristóbal et al., 2018), seguido de los futbolistas masculinos, gimnastas y kayakistas $(54,8 \%, 52,2 \%$ y $51 \%$ respectivamente) (Alacid et al., 2014; Vernetta et al., 2018; López Secanell y Rico Mateu, 2019). Los deportistas con porcentajes medios de ADM por encima del $50 \%$ y que por tanto necesitarían cambiar su alimentación para mejorar estos patrones han sido las jugadoras de futbol femenino del estudio realizado por (González-Neira et al., 2015). Por otro lado, resaltar como negativo que el $58,33 \%$ de las jugadoras de futbol sala, participantes del estudio de Rubio-Arias et al. (2015), presentan una baja adherencia a la DM. Por el contrario, como aspecto positivo, son las Kayistas, gimnastas y los jugadores de futbol masculino los que obtienen mejor adherencia a la DM, (11\%, 5,43\% y 2,5\% respectivamente) (Alacid et al., 2014; Vernetta et al., 2019; López Secanell y Rico Mateu, 2019).

El hecho de que estos deportistas hayan obtenido los mejores índices de ADM es porque todos son adolescentes de élite y quizás estén concienciados de que una nutrición adecuada, además de evitarles problemas de salud, puede incrementar su rendimiento general y darle una mayor ventaja competitiva, así como disminuir el riesgo de sufrir posibles lesiones (Alacid et al., 2014; Rust, 2002). A este respecto, parece que las gimnastas de mayor nivel y edad, son más conscientes de cuidar su peso corporal y apariencia física con una buena alimentación para obtener mejores puntuaciones (Vernetta et al., 2019).

En relación a los diferentes ítems que componen el índice KIDMED, podemos destacar que los mejores resultados han sido hallados en los nadadores, piragüistas y gimnastas (Philippou et al., 2017; Vaquero-Cristóbal et al., 2018; Vernetta et al., 2019), obteniendo los resultados más cercanos al óptimo en el consumo de cereales, aceite de oliva y productos lácteos en el desayuno (Hernández-Camacho et al., 2017; Philippou et al., 2017; Vaquero-Cristóbal et al., 2018; Vernetta et al., 2018). Por otro lado, los peores resultados en las diferentes deportistas han sido no comer más de una pieza de fruta y verdura al día o frutos secos 2 o 3 veces por semana (Alacid et al., 2014; González-Neira et al., 2015; Hernández-Camacho et al., 2017). Respecto a conductas alimentarias negativas como acudir a hamburguesería, consumir bollería y golosinas fueron las jugadoras de fútbol femenino, seguidos de los jugadores de futbol masculino y piragüistas los que mayores abusos presentaban (González-Neira et al., 2015; 
Hernández-Camacho et al., 2017; Vaquero-Cristóbal et al., 2018). El consumo excesivo de comida "rápida" puede resultar ser un inconveniente en la creación de hábitos saludables de cara al futuro, ya que influye en la aparición de enfermedades cardiovasculares, así como, el sobrepeso y la obesidad e incluso puede generar problemas en la memoria y el aprendizaje del adolescente (Pampillo, Arteche y Méndez, 2019).

También destacar como negativo la omisión del desayuno, con resultados similares en las gimnastas de rítmica (12,2\%) y jugadores de futbol (12\%) (Vernetta et al., 2018; Vaquero-Cristóbal et al., 2018) y ligeramente más bajos en kayakistas (7,78\%), piragüistas (7,3\%), (Alacid et al., 2014; González-Neira et al., 2015). El desayuno es considerado como una comida fundamental del día, indicado como una ingesta crucial para obtener unos buenos hábitos saludables (Bacopoulou, Landis, Rentoumis, Tsitsika, y Efthymiou, 2017). De ahí la relevancia de concienciar por parte de los entrenadores a sus respectivos deportistas de la importancia que tiene un buen desayuno, así como la ingesta de productos saludables para garantizar un estado nutricional óptimo que les permita no sólo realizar un entrenamiento adecuado (RubioArias et al., 2015), sino también y lo más importante, prevenir futuros problemas de salud (López-Sobaler, Cuadrado-Soto, Peral-Suárez, Aparicio, y Ortega, 2018).

En cuanto a las medidas antropométricas, se obtuvieron valores normales de IMC, PC y pliegues en las gimnastas de rítmica, tanto en el grupo de niñas como de adolescentes (Vernetta et al., 2018; Vernetta et al., 2019). Igualmente, los estudios realizados en kayakistas o jugadoras de futbol obtuvieron valores normales, aunque ligeramente superiores a las gimnastas, sin encontrar diferencias significativas en función de grado de adhesión de la dieta (Alacid et al., 2014; Rubio-Arias et al., 2015). Estos resultados pueden ser debidos principalmente al componente estético y de gran complejidad técnica que caracteriza a las gimnastas, en el que el IMC y un porcentaje de grasa normales o ligeramente bajos son determinantes, junto a las diversas cualidades físicas básicas, para una buena ejecución y resultado (Aguilera, 2016; Taboada-iglesias, Vernetta y Gutiérrez-Sánchez, 2017) y por otro lado, a la influencia en su rendimiento reportado en kayakistas y jugadores de fútbol (Alacid et al., 2014; López Secanell y Rico Mateu, 2019).

Por otro lado, no existe relación entre los resultados obtenidos a través del índice KIDMED y las variables antropométricas en las kayistas, jugadoras de futbol sala y gimnastas de rítmica (Alacid et al., 2014; Rubio-Arias et al., 2015; Vernetta et al., 2018). Quizás estos datos pueden ser debidos a que las muestras analizadas en cada uno de los estudios, han sido deportistas de élite nacional y todas adolescentes, lo cual ha 
podido provocar una falta de relación, teniendo la mayoría de ellos variables antropométricas similares (Vernetta et al., 2018).

Sin embargo, se encuentra correlaciones entre IMC y la ADM en jugadores de fútbol masculino y gimnastas de rítmica ya que abarcan rangos de edades que implican tanto a niñas, preadolescente y adolescentes (López Secanell y Rico Mateu, 2019; Vernetta et al., 2019).

\section{CONCLUSIONES}

Como conclusiones, se puede destacar que la mayoría de los estudios incluidos en esta revisión obtienen una alta calidad tras el análisis de las directrices STROBE, no encontrándose ningún estudio con baja calidad según estas directrices. Los adolescentes que practican piragüismo, fútbol masculino, gimnasia rítmica y kayak obtienen los mejores resultados en cuanto a la óptima ADM. Sin embargo, los patrones alimentarios de ADM son muy mejorables en la mayoría de los adolescentes practicantes de las diversas disciplinas deportivas, siendo más pronunciado en las jugadoras de fútbol femenino.

Los resultados más cercanos al óptimo fueron el consumo de aceite para cocinar, cereales para desayunar y el consumo de fruta y verdura una vez al día. Por el contrario, como negativo se destaca el abuso de acudir más de una vez a la semana a la hamburguesería sobre todo en jugadores de futbol femenino y masculino y en piragüistas.

No existe una relación entre la ADM con las medidas antropométricas realizadas en adolescentes practicantes de kayak, fútbol sala y gimnasia, pero si existe esta asociación entre niños y adolescentes practicantes de fútbol y gimnasia rítmica al tener rangos de edades superiores.

Como principales limitaciones de la presente revisión son los escasos estudios que hay en general sobre la $A D M$ en deportistas de cualquier modalidad deportiva. Además, el $90 \%$ de los estudios son trasversales, encontrándose únicamente un estudio longitudinal, aspecto que podría ser considerado para posteriores investigaciones sobre la ADM y conocer cómo evoluciona la ADM en los deportistas durante un periodo de tiempo prolongado. Por otro lado, gran parte de los estudios abarcan a deportistas que se encuentran en diferentes niveles competitivos, así como etapas de crecimiento y de género diferente, por lo que quizás ha sido complicado realizar comparativas entre ello. No obstante, los deportistas adolescentes con franja de edades 14-17 años suelen tener mejores resultados que los preadolescentes (López Secanell y Rico Mateu, 2019; Manzano-Carrasco et al., 2020). 
Por tanto, dada la efectividad de DM sobre sus conocidos beneficios para la salud según Sofi, Cesari, Abbate, Franco, y Casini (2008), es necesario potenciar su consumo teniendo especial atención en estos preadolescentes donde parece existir una clara tendencia al rápido abandono del patrón dietético mediterráneo (Fernández San Juan, 2006).

\section{REFERENCIAS}

*Estudios tomados en cuenta para la revisión

Aguilera, F. L. (2016). Estado nutricional, satisfacción con la imagen corporal e ingesta alimentaria en adolescentes que practican Gimnasia Artística (Tesis de licenciatura). Universidad FASTA, Argentina. Recuperado de http://redi.ufasta.edu.ar:8080/xmlui/handle/123456789/1272

Akar, S., y Sanlier, N. (2011). Compliance with Mediterranean Diet Quality Index (KIDMED) and nutrition knowledge levels in adolescents. A case study from Turkey. Appetite, 57(1), 272-277. doi: https://doi.org/10.1016/j.appet.2011.05.307

*Alacid, F., Vaquero-Cristóbal, R., Sánchez-Pato, A., Muyor, J.M., y López-Miñarro, P. Á. (2014). Adhesión a la dieta mediterránea y relación con los parámetros antropométricos de mujeres jóvenes kayakistas. Nutrición Hospitalaria, 29(1), 121-127. doi: https://doi.org/10.3305/nh.2014.29.1.6995

Allen, B., y Waterman, H. (2019). Etapas de la adolescencia. Recuperado de https://www.healthychildren.org/Spanish/ages-stages/teen/Paginas/Stages-ofAdolescence.aspx

Ayechu, A., y Durá, T. (2009). Dieta mediterránea y adolescentes. Nutrición Hospitalaria, 24(6), 751-762. doi: https://doi.org/10.3305/nh.2009.24.6.4567

Ayechu, A., y Durá, T. (2010). Calidad de los hábitos alimentarios (adherencia a la dieta mediterránea) en los alumnos de educación secundaria obligatoria. Anales del Sistema Sanitario de Navarra, 33(1), 35-42. Recuperado de http://scielo.isciii.es/pdf/asisna/v33n1/original3.pdf

Bacopoulou, F., Landis, G., Rentoumis, A., Tsitsika, A., y Efthymiou, V. (2017). Mediterranean diet decreases adolescent waist circumference. European Journal of Clinical Investigation, 47(6), 447-455. doi: https://doi.org/10.1111/eci.12760

Cabrero, M., García, A., Salinero, J.J., Pérez, B., Sánchez, J.J., y García, R., Robledo, S., y lbáñez, R. (2012). Calidad de la dieta y su relación con el IMC y el sexo en adolescentes. Revista Española de Nutrición Clínica y Dietética Hospitalaria, 32(2), 21-27. Recuperado de 
https://www.researchgate.net/publication/288284049 Diet quality and its relati on to sex and BMI in adolescents

Córdoba-Caro, L.G., Luego, L.M., y García, V. (2012). Adecuación nutricional de la ingesta de los estudiantes de secundaria de Badajoz. Nutrición Hospitalaria, 27(4), 1065-1071. doi: https://doi.org/10.3305/nh.2012.27.4.5800

Donini, L.M., Serra-Majem, L., Bulló, M., Gil, A., y Salas-Salvadó, J. (2015). The Mediterranean diet: culture, health and science. The British Journal of Nutrition, 113(S2), 51-53. doi: https://doi.org/10.1017/S0007114515001087

Durá-Travé, T., Gallinas-Victoriano, F., Mosquera-Gorostidi, A., Justo-Ranera, A., y Martín-García, I. S. (2014). Adherence to mediterranean diet in students of compulsory secondary education. In Focus on Nutrition in Child-Juvenile Populations in a Mediterranean Area (pp. 15-26). United States of America: NOVA

Dussaillant, C., Echevarría, G., Urquiaga, I., Velasco, N., y Rigotti, A. (2016). Evidencia actual sobre los beneficios de la dieta mediterránea en salud. Revista Médica de Chile, 144(8), 1044-1052. doi: http://dx.doi.org/10.4067/S0034$\underline{98872016000800012}$

Fernández San Juan, P. M. (2006). Dietary habits and nutritional sta- tus of school aged children in Spain. Nutrición Hospitalaria, 21(3), 374-378. Recuperado de https://pubmed.ncbi.nlm.nih.gov/16771121/

*González-Neira, M., Mauro-Martín, I., García-Aguado, B., Fajardo, D., y Garicano-Vilar, E. (2015). Valoración nutricional, evaluación de la composición corporal y su relación con el rendimiento deportivo en un equipo de fútbol femenino. Revista Española de Nutrición Humana y Dietética, 19(1), 36-48. doi: https://doi.org/10.14306/renhyd.19.1.109

*Hernández-Camacho, J. D., Fuentes-Lorca, E., y Moya-Amaya, H. (2017). Características antropométricas, somatotipo y patrones alimentarios en jugadores jóvenes de fútbol. Revista Andaluza de Medicina Del Deporte, 10(4), 192-196. doi: http://dx.doi.org/10.1016/j.ramd.2017.01.004

Idelson, P. I., Scalfi, L., y Valerio, G. (2017). Adherence to the Mediterranean Diet in children and adolescents: A systematic review. Nutrition, Metabolism and Cardiovascular Diseases, 27(4), 283-299. doi: https://doi.org/10.1016/j.numecd.2017.01.002

*López Secanell, I., y Rico Mateu, R. (2019). La adhesión a la dieta mediterránea en los jugadores de las categorías inferiores de un club de futbol de alto rendimiento y su relación con el índice de masa corporal. TRANCES: Revista de Transmisión del Conocimiento Educativo y de la Salud, 11(4), 553-570. Recuperado de https://dialnet.unirioja.es/servlet/articulo?codigo $=7080313$ 
López-Sobaler, A.M., Aparicio, A., y Ortega, R. M. (2017). Papel del huevo en la dieta de deportistas y personas físicamente activas. Nutrición Hospitalaria, 34(4), 1-71. Recuperado de

https://www.nutricionhospitalaria.org/index.php/articles/01568/show

López-Sobaler, A.M., Cuadrado-Soto, E., Peral-Suárez, Á., Aparicio, A., y Ortega, R. M. (2018). Importancia del desayuno en la mejora nutricional y sanitaria de la población. Nutrición Hospitalaria, 35(6), 3-6. doi: http://dx.doi.org/10.20960/nh.2278

Lynch, C., Kristjansdottir, A.G., Te Velde, S.J., Lien, N., Roos, E., Thorsdottir, I. ... Yngve, A. (2014). Fruit and vegetable consumption in a sample of 11-year-old children in ten European countries - the PRO GREENS cross-sectional survey. Public Health Nutrition, 17(11), 2436-2444. doi: https://doi.org/10.1017/s1368980014001347

*Manzano-Carrasco, S., Felipe, J.L., Sánchez-Sánchez, J., Hernández-Martín, A., Clavel, I., Gallardo, L., y García-Unanue, J. (2020). Relationship between Adherence to the Mediterranean Diet and Body Composition with Physical Fitness Parameters in a Young Active Population. International Journal of Enviromental Research and Public Health, 17(9), e3337. doi: https://doi.org/10.3390/ijerph17093337

Márquez, S. (2008). Trastornos alimentarios en el deporte: factores de riesgo, consecuencias sobre la salud, tratamiento y prevención. Nutrición Hospitalaria, 23(3), 183-190. Recuperado de http://scielo.isciii.es/pdf/nh/v23n3/revision1.pdf

Márquez-Sandoval, F., Bulló, M., Vizmanos, B., Cass-Agustench, P., y Salas-Salvado, J. (2008). Un patrón de alimentación saludable: la dieta mediterránea tradicional. Antropo, 16, 11-22. Recuperado de https://bibliotecavirtual.diba.cat/documents/10934/3667829/document1.pdf

Martín, L., Segura, O., Lozano, M., Navarro, A., Ortega, R., y López-Sobaler, A. (2004). Estudio de los hábitos alimenticios de un colectivo de escolares de la Comunidad de Madrid. V Congreso de la Sociedad Española de Nutrición Comunitaria. Madrid. Martínez-González, M. Á., De la Fuente-Arrillaga, C., Nunez-Cordoba, J. M., BasterraGortari, F. J., Beunza, J. J., Vazquez, Z. ... Bes-Rastrollo, M. (2008). Adherence to Mediterranean diet and risk of developing diabetes : prospective cohort study. British Medical Journal, 336(7657), 1348-1351. doi: https://doi.org/10.1136/bmj.39561.501007.BE

Moher, D., Liberati, A., Tetzlaff, J., y Altman, D. (2009). Preferred reporting items for systematic reviews and meta-analyses: the PRISMA statement. BMJ, 339, 332336. doi: https://doi.org/10.1136/bmj.b2535 
Moreno, M.A., y Ortiz, G. R. (2009). Trastorno alimentario y su relación con la imagen corporal y la autoestima en adolescentes. Terapia Psicológica, 27(2), 181-190. doi: http://dx.doi.org/10.4067/S0718-48082009000200004

Pampillo, T., Arteche, N., y Méndez, M. A. (2019). Hábitos alimentarios, obesidad y sobrepeso en adolescentes de un centro mixto. Revista de Ciencias Médicas de Pinar Del Río, 23(1), 99-107. Recuperado de https://www.medigraphic.com/pdfs/pinar/rcm-2019/rcm191n.pdf

*Philippou, E., Middleton, N., Pistos, C., Andreou, E., y Petrou, M. (2017). The impact of nutrition education on nutrition knowledge and adherence to the Mediterranean Diet in adolescent competitive swimmers. Journal of Science and Medicine in Sport, 20(4), 328-332. doi: https://doi.org/10.1016/j.jsams.2016.08.023

*Rubio-Arias, J.Á., Ramos, D.J., Ruiloba, J.M., Carrasco, M., Alcaraz, P.E., y Jiménez, F. J. (2015). Adhesión a la dieta mediterránea y rendimiento deportivo en un grupo de mujeres deportistas de élite de fútbol sala. Nutrición Hospitalaria, 31(5), 22762282. doi: https://doi.org/10.3305/nh.2015.31.5.8624

Rust, D. M. (2002). The female athlete triad: Disordered eating, amenor- rhea, and osteoporosis. The Clearing House: A Journal of Educational Satrategies, Issues and Ideas, 75(6), 301-305. doi: https://doi.org/10.1080/00098650209603960

Sánchez-Cruz, J.J., Jiménez-Moleón, J.J., Fernández-Quesada, F., y Sánchez, M.J. (2013). Prevalencia de obesidad infantil y juvenil en España en 2012. Revista Española de Cardiología, 66(5), 371-376. doi: 10.1016 / j.recesp.2012.10.016

Santomauro, F., Lorini, C., Tanini, T., Indiani, L., Lastrucci, V., Comodo, N., y Bonaccorsi, G. (2014). Adherence to Mediterranean diet in a sample of Tuscan adolescents. Nutrition, 30(11-12), 1379-1383. doi: https://doi.org/10.1016/j.nut.2014.04.008

Sawyer, S.M., Afifi, R.A., Bearinger, L.H., Blakemore, S.J., Dick, B., y Ezeh, A.C. (2012). Adolescence: a foundation for future health. The Lancet, 379(9826), 1630-1640. doi: https://doi.org/10.1016/S0140-6736(12)60072-5

Sawyer, S.M., Azzopardi, P.S., Wickremarathne, D., y Patton, G. C. (2018). La edad de la adolescencia. The Lancet Child \& Adolescent Health, 2(3), 223-228. doi: https://doi.org/10.1016/s2352-4642(18)30022-1

Serra-Majem, L., García-Closas, R., Ribas, L., Pérez-Rodrigo, C., y Aranceta, J. (2001). Food patterns of Spanish schoolchildren and adolescents: The enKid Study. Public Health Nutrition, 4(6A), 1433-1438. doi: https://doi.org/10.1079/phn2001234

Sofi, F., Cesari, F., Abbate, R., Franco, G., y Casini, A. (2008). Adherencia a la dieta mediterránea y estado de salud: metanálisis. BMJ, 337. doi: https://doi.org/10.1136/bmj.a1344 
Strong, W.B., Malina, R.M., Blimkie, C.J.R., Daniels, S.R., Dishman, R.K., Gutin, B., Hergenroeder, A.C. ... Trudeau, F. (2005). Evidence based physical activity for school-age youth. The Journal of Pedriatrics, 146(6), 732-737. doi: https://doi.org/10.1016/i.jpeds.2005.01.055

Sundgot-Borgen, J., y Torstveit, M. K. (2004). Prevalence of eating disorders in elite athletes is higher than in the general population. Clinical Journal of Sport Medicine, 14(1), 25-32. doi: https://doi.org/10.1097/00042752-200401000-00005

Taboada-Iglesias, Y., Vernetta, M., y Gutiérrez-Sánchez, Á. (2017). Anthropometric Profile in Different Event Categories of Acrobatic Gymnastics. Journal of Human Kinetics, 57, 169-179. Recuperado de https://www.ncbi.nlm.nih.gov/pmc/articles/PMC5504589/

*Vaquero-Cristóbal, R., Alacid, F., Muyor, J.M., y López-Miñarro, P. A. (2018). Relación entre los parámetros antropométricos y la adhesión a la dieta mediterránea en jóvenes piragüistas hombres de élite. MHSalud, 15(2), 20-35. doi: https://doi.org/10.15359/mhs.15-2.2

*Vernetta, M., Montosa, I., Ariza, L., y López Bedoya, J. (2019a). Comparative analysis of adherence to the mediterranean diet among girls and adolescents who perform rhythmic gymnastics. Revista Brasileira de Medicina do Esporte, 25(4), 280-284. doi: http://dx.doi.org/10.1590/1517-869220192504175283

Vernetta, M., Montosa, I., y López-Bedoya, J. (2018). Dieta Mediterránea en jóvenes practicantes de gimnasia rítmica. Revista Chilena de Nutrición, 45(1), 37-44. doi: http://dx.doi.org/10.4067/s0717-75182018000100037

*Vernetta Santana, M., Peláez, E.M., Ariza, L., y López-Bedoya, J. (2019b). Dieta mediterránea, estima corporal y parámetros antropométricos en adolescentes practicantes de gimnasia acrobática [Ahead of print]. Revista Mexicana de Trastornos Alimentarios, 10(2). Recuperado de http://journals.iztacala.unam.mx/index.php/amta/pages/view/avanceslinea

Zapico, A., Blández, J., y Fernández, E. (2010). Sobrepeso, obesidad y adecuación a la dieta mediterránea en adolescentes de la comunidad de Madrid. Archivos de Medicina Del Deporte, 27(138), 271-280. Recuperado de http://femede.es/documentos/Original Sobrepeso obesidad 271 138.pdf 